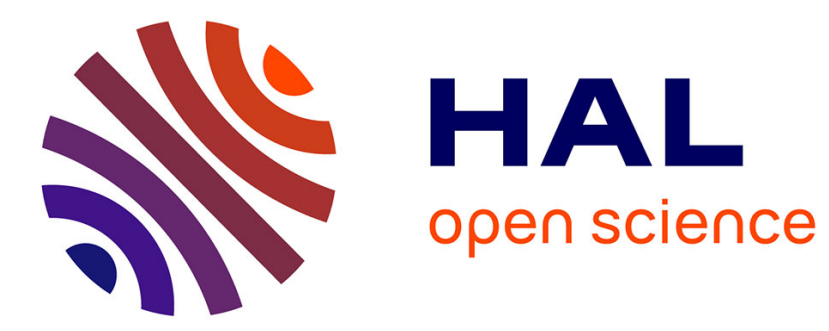

\title{
Probabilistic description of the electron motion in the Coulomb field of nucleus
}

\author{
M. Gryziński
}

\section{To cite this version:}

M. Gryziński. Probabilistic description of the electron motion in the Coulomb field of nucleus. Journal de Physique Lettres, 1982, 43 (12), pp.425-430. 10.1051/jphyslet:019820043012042500 . jpa00232071

\section{HAL Id: jpa-00232071 https://hal.science/jpa-00232071}

Submitted on 1 Jan 1982

HAL is a multi-disciplinary open access archive for the deposit and dissemination of scientific research documents, whether they are published or not. The documents may come from teaching and research institutions in France or abroad, or from public or private research centers.
L'archive ouverte pluridisciplinaire HAL, est destinée au dépôt et à la diffusion de documents scientifiques de niveau recherche, publiés ou non, émanant des établissements d'enseignement et de recherche français ou étrangers, des laboratoires publics ou privés. 


\title{
Probabilistic description of the electron motion in the Coulomb field of nucleus
}

\author{
M. Gryziński \\ Institute of Nuclear-Research, Swierk/Otwock, Poland
}

(Reçu le 17 novembre 1981, révisé le 22 mars 1982, accepté le 20 avril 1982)

\begin{abstract}
Résumé. - Cet article établit, pour un électron d'une orbite de Kepler de moment cinétique fixé, l'expression d'une probabilité de localisation spatiale, ainsi qu'une probabilité de distribution en vitesses. Les expressions obtenues résultent des équations classiques du mouvement. Pour l'espace des impulsions, la probabilité de l'ensemble microcanonique des orbites de Kepler s'avère ainsi correspondre à la probabilité quantique. Des arguments sont exposés sur l'intérêt de la distribution dite « de chute libre » dans une théorie classique cohérente des chocs binaires.
\end{abstract}

\begin{abstract}
In this paper radial localization probability and probability in velocity space (velocity distribution function) for an electron staying in the Kepler orbit of specified angular momentum (of specified eccentricity) were derived from classical equations of motion. Probabilities for a microcanonical set of Kepler orbits and for a zero angular momentum free-fall orbit were also calculated. It is pointed out that from the point of view of consistency of classical theory the free-fall distribution should be used in binary encounter description of atomic collisions.
\end{abstract}

Since 1959, when some basic relations for the two-body collision in the laboratory system of coordinates were derived and successfully applied to a quantitative description of various inelastic atomic collisions [1], the binary encounter approach became a widely used method of atomic collision analysis [2-7]. The atomic cross section, which is the fundamental quantity of any atomic collision theory, is defined in this approach in the following way :

$$
\sigma^{\mathrm{at}}=\int \sigma^{\text {b.e. }}\left(v_{\mathrm{e}}\right) \cdot f^{\mathrm{at}}\left(v_{\mathrm{e}}\right) \mathrm{d} v_{\mathrm{e}},
$$

where $\sigma^{\text {b.e. }}\left(v_{\mathrm{e}}\right)$ is the binary cross section for a collision with an atomic electron of velocity $v_{\mathrm{e}}$ and $f^{\text {at }}\left(v_{e}\right)$ is the electron velocity distribution function. The latter describes the behaviour of electrons in the target atom (molecule). Since the behaviour of electrons in different atoms (molecules) is different, the electron velocity function $f^{\text {at }}\left(v_{\mathrm{e}}\right)$ should be in each of the investigated cases specified separately. Sometimes, however, the details of the motion are unimportant and approximate description of the shell may be satisfactory. Particularly in different binary encounter calculations the hydrogen-like velocity distribution function can be used. This function can be 
easily derived from the well known relation describing the electron motion in the Coulomb field of nucleus :

$$
t=\frac{T}{2 \pi}\left\{\arccos \left[\frac{1}{\varepsilon}\left(1-\frac{r}{a}\right)\right]-\sqrt{\varepsilon^{2}-\left(1-\frac{r}{a}\right)^{2}}\right\}+\text { const. }
$$

where $a$ is the large semi-axis of the ellipse

$$
a=\frac{z_{\mathrm{e}}^{2}}{2|E|}
$$

$\varepsilon$ is the eccentricity of the ellipse

$$
\varepsilon=\sqrt{1-\frac{2|E|}{m}\left(\frac{L}{z_{\mathrm{e}}^{2}}\right)^{2}}
$$

$T$ is the period of motion

$$
T=\pi z_{\mathrm{e}}^{2} \sqrt{\frac{m}{2|E|^{3}}}
$$

and $L$ and $E$ are two constants of motion.

The first of the constants represents the angular momentum

$$
L=m r^{2} \dot{\varphi}=\text { const. }
$$

and the second one - the binding energy of the electron

$$
E=\frac{m v^{2}}{2}-\frac{z_{\mathrm{e}}^{2}}{r}=\text { const. }
$$

A classical description of atomic collision experiments corresponds to the average of many collisions with atoms in different moments of the electron orbital motion :

$$
\bar{\sigma}=\frac{1}{T} \int_{0}^{T} \sigma(t) \mathrm{d} t
$$

In the binary encounter approximation, relations depend on time only indirectly - through electron orbital velocity $v_{\mathrm{e}}(t)$. It is convenient, therefore, to introduce $v_{\mathrm{e}}$ as the independent variable.

In this case equation (8) can be written in the following way :

$$
\bar{\sigma}=\int \sigma^{\text {b.e. }}\left(v_{\mathrm{e}}\right)\left(\frac{1}{T\left(\mathrm{~d} v_{\mathrm{e}} / \mathrm{d} t\right)}\right) \mathrm{d} v_{\mathrm{e}}
$$

where $1 / T .\left(\mathrm{d} v_{\mathrm{e}} / \mathrm{d} t\right)$ is the electron velocity distribution function. In the investigated case the latter can be easily derived from equations (2) and (7) by simple differentiation. Introducing the dimensionless velocity

$$
u=\left(v_{\mathrm{e}} / v_{\mathrm{E}}\right)
$$

with $v_{E}$ given by

$$
v_{E}=\sqrt{2|E| / m},
$$


one can show that

$$
\bar{\sigma}=\int \sigma^{\text {b.e. }}(u) \cdot p(u, \varepsilon) \mathrm{d} u
$$

where

$$
p(u, \varepsilon)=\frac{4}{\pi} \frac{1}{\left(1+u^{2}\right)^{2}} \frac{1}{\sqrt{1-\left(\frac{1-u^{2}}{2 u}\right)^{2}\left(1-\varepsilon^{2}\right)}} .
$$

The function $p(u, \varepsilon)$ represents the probability that the velocity of the electron staying on the elliptic orbit of eccentricity $\varepsilon$ is contained between $u$ and $u+\mathrm{d} u$. For the free-fall (f.f.) zero angular momentum orbit $(\varepsilon=1)$ the velocity distribution function $p(u)$ assumes the form

$$
p^{\mathrm{f.f}}(u)=\frac{4}{\pi} \frac{1}{\left(1+u^{2}\right)^{2}}
$$

In the atom there is a discrete set of stable orbits. In the case of hydrogen atom those are defined by the two sets of integers : $n$ and $l$, which specify the electron energy and angular momentum :

$$
\begin{aligned}
& E_{n}=-\frac{z^{2} e^{4} m}{2 n^{2} \hbar^{2}}, \\
& L_{l}=l \hbar .
\end{aligned}
$$

In such a case :

$$
\varepsilon=\sqrt{1-\left(\frac{l}{n}\right)^{2}}
$$

and the velocity distribution function assumes the form :

$$
p_{n, l}(u)=\frac{4}{\pi} \frac{1}{\left(1+u^{2}\right)^{2}} \frac{1}{\sqrt{1-\left(\frac{1-u^{2}}{2 u}\right)^{2}\left(\frac{l}{n}\right)^{2}}} .
$$

Sometimes one can be interested in the collision with an atom which is just emitting the quantum, then the values of $L$ and $E$ are somewhere between those determined by the integers $n$ and $l$. Then the velocity distribution function as given by equation (13) must be used.

Using this equation, it is possible to determine cross section for collisions with atoms which have just emitted the specified « fraction " of the quantum and therefore within the binary encounter collision theory to describe collisional broadening of spectral lines (the paper concerning this point will be published in not very far future).

If a charged particle penetrates the gaseous medium formed by Kepler systems of different binding energies and angular momentum (i.e. of different eccentricities) the effective cross section is given by :

$$
\sigma_{\text {eff }}=\iint \bar{\sigma}(L, E) g(L, E) \mathrm{d} L \mathrm{~d} E,
$$

where $g(L, E)$ is the population function describing the state of the medium. 
For a particular case of the ensemble of Kepler orbits with population as given below

$$
g(L, E)=\left(\frac{2}{L_{\max }^{2}}\right) \cdot L \text { and } 0 \leqslant L \leqslant L_{\max }=\text { const. }
$$

which is called a microcanonical distribution, if only binary encounter cross section does not depend directly on angular momentum of the target system electron, one will have :

$$
\sigma_{\text {eff }}^{\text {micr }}=\int \bar{\sigma}(u)\langle p(u)\rangle^{\text {micr }} \mathrm{d} u,
$$

where $\langle p(u)\rangle^{\text {micr }}$ represents the average for orbits of the same $E$ and of different $L$ (i.e. of different eccentricities)

$$
\langle p(u)\rangle^{\text {micr }}=\frac{4}{\pi} \frac{1}{\left(1+u^{2}\right)^{2}} \int_{0}^{L_{\max }}\left(\frac{2}{L_{\max }^{2}}\right) \frac{L \mathrm{~d} L}{\sqrt{1-\left(\frac{1-u^{2}}{2 u}\right)^{2}\left(\frac{L}{L_{\max }}\right)^{2}}} .
$$

Performing integration, one finds that

$$
\langle p(u)\rangle^{\text {micr }}=\frac{4}{\pi} \frac{8 u^{2}}{\left(1+u^{2}\right)^{4}} .
$$

This is the distribution which, being called the microcanonical distribution, is the same as given by quantum mechanics for the target momentum density and which is commonly used for description of the ground-state atomic system in atomic collisions calculations, including classical calculations (see for instance Abrines and Percival [8]).

There are atomic collision processes which depend upon localization of the collision between the projectile and atomic electron with respect to the nucleus : inner shell ionization by heavy charged particles correlated with the deflection of the ionizing particle in the field of nucleus is among them. To describe the processes of this type within b.e.a., it is necessary to know the radial localization probability. The latter can be easily derived from equation (8) taking into account that in orbital motion $t$ and $r$ are given by equation (2). In result, we obtain :

$$
p(\rho)=\frac{1}{\pi} \frac{\rho^{2}}{\sqrt{-\rho^{2}+\rho-4\left(1-\varepsilon^{2}\right)}},
$$

where $\rho$ is the distance from nucleus in relative units :

$$
\rho=\frac{r}{2 a}
$$

For the free-fall orbit and for microcanonical distribution function the radial localization probabilities respectively are :

$$
\begin{aligned}
p^{\text {f.f. }}(\rho) & =\frac{1}{\pi} \sqrt{\frac{\rho}{1-\rho}}, \\
\langle p(\rho)\rangle^{\text {micr }} & =\frac{8}{\pi} \sqrt{\rho^{3}(1-\rho)} .
\end{aligned}
$$

The derived probability functions are shown in figures 1 and 2. 


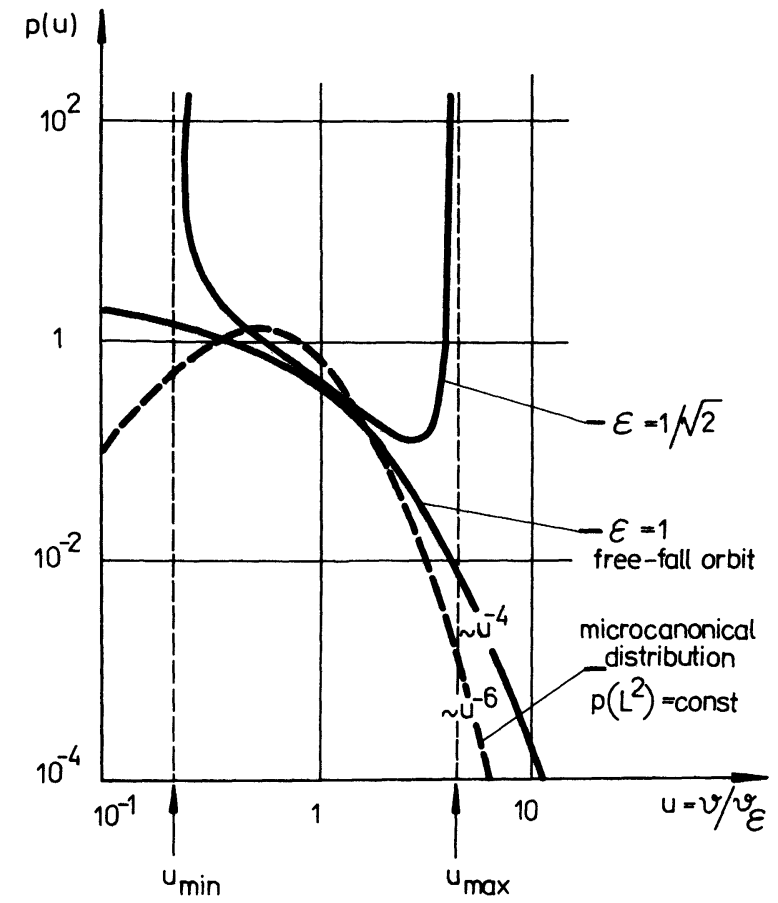

Fig. 1. - Velocity distribution functions for two particular Kepler orbits $(\varepsilon=1 / \sqrt{2}$ and 1$)$ and for a microcanonical set of Kepler orbits.

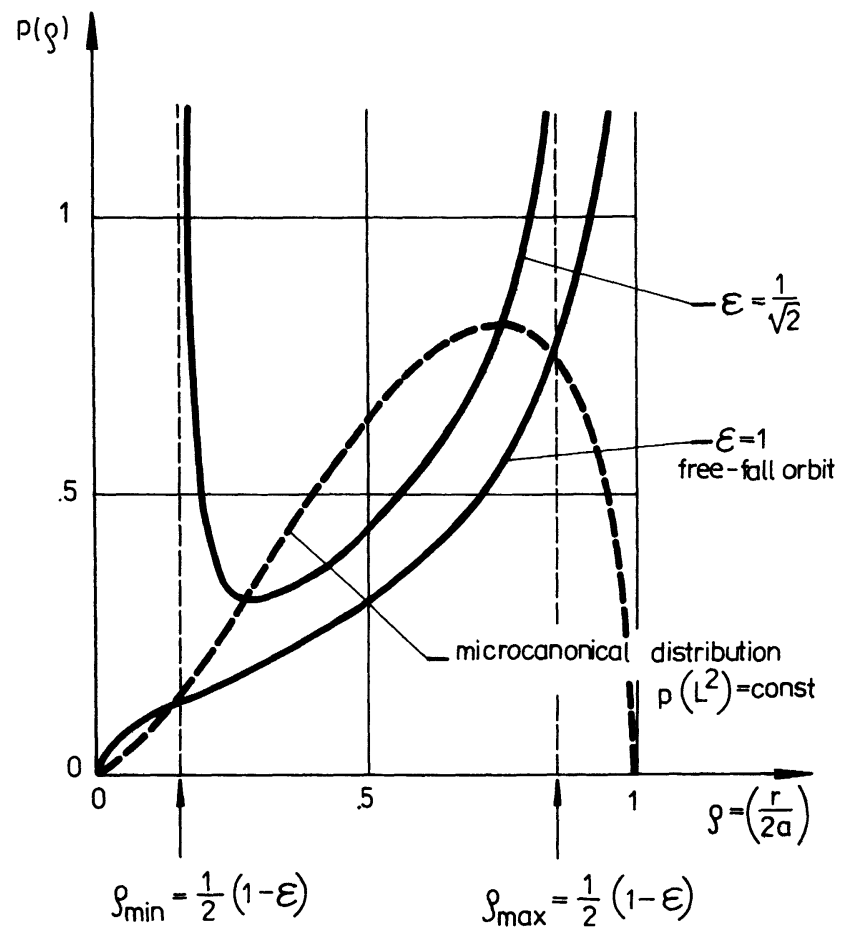

Fig. 2. - Radial localization probability for two particular Kepler orbits $(\varepsilon=1 / \sqrt{2}$ and 1$)$ and for a microcanonical set of Kepler orbits. 
At the end, it is important to note that the use of $\langle p(u)\rangle^{\text {micr }}$ and $\langle p(\rho)\rangle^{\text {micr }}$, obtained in result of averaging over $L$, for description of the ground-state atom is from the point of view of consistency of classical approach inadmissible. The behaviour of electrons in identical atoms should be identical and, therefore, not only binding energies but angular momenta as well should be identical. This means that the velocity distribution function $p(u)$ and the density distribution function $p(\rho)$ as given by equations (14) and (23) should be used in classical atomic collision calculations for description of the ground-state hydrogen atom and hydrogen-like ions [9].

In the case of many electron atoms, one arrives to a similar conclusion $-\langle p(u)\rangle^{\text {micr }}$ and $\langle p(\rho)\rangle^{\text {micr }}$ cannot represent the atom in classical atomic collision calculations [10].

The answer to the question : what velocity distribution and radial localization probability can represent the atom ? - may only be found by comparing the results of consistent collision theory with the experimental data.

Recent progress in the technique of atomic collision experiments, particularly development of high energy electron Compton scattering $[11,12]$ assorted with $(e, 2 \mathrm{e})$ coincidence measurements and inner shell ionization by protons correlated with deflection of the latter in the field of nucleus $[13,14]$ form grounds for precise answering of this important question of atomic physics.

\section{References}

[1] GryZiński, M., Phys. Rev. 115 (1959) 374.

[2] Stabler, R. C., Phys. Rev. 133A (1964) 1268.

[3] Gryziński, M., Phys. Rev. 138A (1965) 336.

[4] Delcroix, J. L., Physique des plasmas (Dunod, Paris) 1966.

[5] VRIENS, L., Case studies in atomic collision physics, ed. by E. W. Mc Daniel and M. R. C. Mc Dowell, (North Holland, Amsterdam) 1969.

[6] BATES, D. R., Phys. Rep. 35 (1978) 307.

[7] Gryziński, M., J. Physique Colloq. 40 (1979) C7-171.

[8] Abrines, R. and Percival, J. C., Proc. Phys. Soc. 88 (1966) 861.

[9] Gryziński, M., Kunc, J., Zgorzelski, M., J. Phys. B 6 (1973) 2292.

[10] Gryziński, M., OKopińska, A., Proc. of the VIII ZCPEAC, Beograd, 1973, p. 635.

[11] Barlas, A. D., Rueckner, W. H. E. and Wellenstein, H. F., J. Phys. B. 11 (1978) 3381.

[12] Bennani, A. L., Duguet, A. and Wellenstein, H. F., J. Phys. B. 12 (1979) 461.

[13] Hansen, J. S., Phys. Rev. 8A (1973) 822.

[14] Laegsgard, E., Andersen, J. U. and Feldman, L. C., Phys. Rev. Lett. 29 (1972) 1206. 